\title{
Management of a Cluster of Foreign Body Ingestion Incidents in Patients with Borderline Personality Disorder
}

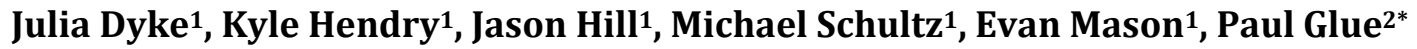 \\ ${ }^{1}$ Southern District Health Board and University of Otago, Dunedin, New Zealand \\ ${ }^{2}$ Department of Psychological Medicine, Dunedin School of Medicine, University of Otago, Dunedin, \\ New Zealand \\ Email: *paul.glue@otago.ac.nz
}

Received 10 December 2013; revised 10 January 2014; accepted 17 January 2014

Copyright (C) 2014 by authors and Scientific Research Publishing Inc.

This work is licensed under the Creative Commons Attribution International License (CC BY). http://creativecommons.org/licenses/by/4.0/

(c) (i) Open Access

\begin{abstract}
Objective: Deliberate foreign body ingestion (DFBI) has been reported in patients with personality disorders as part of a spectrum of self-harming behaviours, however the published literature is small (15 case reports). Method: This was a retrospective audit of medical and psychological management of a cluster of 12 DFBI incidents over 9 months in 4 young female patients with Borderline Personality Disorder (BPD). Results: All four patients knew one another from outpatient psychotherapy programmes. DFBI was associated with substance use in 5/12 incidents, and with precipitants (experiencing strong emotions, witnessing self-harm) in 4/12 incidents. Most DFBI involved sharp objects (razor blades, glass, safety pins). Medical intervention was initially active (endoscopy/surgery) but progressed to a more conservative, observational approach. Psychological management was initially restrictive, but also changed over time to focus on identifying distress, enhancing coping mechanisms and patient responsibility. Co-ordination of patient care management involved intensive liaison (provision of interdisciplinary support and education) between medical, surgical and psychiatric teams. Conclusion: Management of DFBI in patients with BPD requires high levels of interdisciplinary collaboration. In this cluster of DFBI incidents, effective management was achieved with conservative medical/surgical methods (observation) and non-restrictive psychological approaches that enhanced patient self-efficacy.
\end{abstract}

\section{Keywords}

Borderline Personality Disorder, Swallowing, Foreign Body, Clinical Management

\footnotetext{
${ }^{*}$ Corresponding author.
} 


\section{Introduction}

Ingestion of foreign bodies is in many cases accidental, and may occur in young children, patients undergoing dental surgery, in bulimic patients (who may swallow an object used to induce emesis), and in intoxicated individuals [1]. It differs from deliberate ingestion of foreign bodies (DFBI), which is associated with high rates of psychiatric diagnoses and is frequently repetitive [2]. Recent review articles have identified several clinical/etiological subgroups within patients with DFBI, psychosis, malingering, pica or obsessive-compulsive disorder, and borderline personality disorder [1] [3]. DFBI has also been noted in prison populations, however it appears to reflect malingering, rather than underlying mental health problems [3].

To date, there are 15 case reports of DFBI in patients with personality disorders (Table 1), comprising mainly young females, most of whom have Borderline Personality Disorder (BPD). However, based on clinical anecdotes, DFBI in BPD may be more prevalent than the small number of case reports might indicate [3] [4]. Management of DFBI in BPD patients in medical and surgical settings is challenging, as it is often repetitive, and the potential for further self-harm and complex emotional issues may elicit strong countertransference from nursing and medical staff. There are well-established guidelines for acute medical and surgical management of foreign body ingestion [5] [6], however none on the appropriateness and timing of repeated endoscopies for repeated DFBI [4], and none of the guidelines consider the role of secondary gain or reinforcement in strategies for managing these patients. The objective of this paper is to report an audit of a cluster of DFBI in 4 patients with BPD, along with the evolution of medical, surgical and psychiatric management strategies during this time.

\section{Methods}

This was a retrospective audit of a cluster of 12 incidents of DFBI in 4 female patients with Borderline Personality Disorder at a single hospital in Dunedin, New Zealand, over a 9 month period. Audits deemed to be minimal risk observational studies are exempted from ethics committee review in New Zealand [7]. The audit was initiated following a number of interdisciplinary meetings between Medical, Surgical and Mental Health teams, in response to management issues around these patients. Patients' medical files, contacts with clinical services, and contacts with one another were reviewed, and possible precipitant associated with DFBI (e.g. intoxication, stressors) were recorded. Changes in management strategies for medical, surgical and psychological teams over time were reviewed.

\section{Results and Discussion}

Patients: The four patients were female, age range 19 - 22. All had primary diagnoses of DSM-5 Borderline Personality Disorder [8]. All had extensive histories of contact with mental health services, and all had multiple prior episodes of self-harm including cutting and overdosing. All four patients knew one another and were attending the same outpatient psychotherapy programmes. Additionally two patients were hospitalized on the same inpatient ward for several months.

DFBI Incidents: The chronology of the 12 DFBI incidents is shown in Table 2. Eight incidents involved swallowing of razor blades, and there were individual incidents of swallowing sewing pins, glass fragments, pencils and a toothbrush, and a blade from a vegetable peeler along with broken glass. The intent of DFBI was non-lethal: one patient described it as “cutting from the inside”. In all cases, DFBI was either reported to nursing or medical staff, or patients reported abdominal discomfort or other symptoms which led to disclosure of recent DFBI. DFBI was associated with prior substance use in 5/12 incidents (most commonly synthetic cannabis), and with precipitants (experiencing strong emotions related to feelings of rejection or helplessness, witnessing self-harm) in 4/12 incidents. The DFBI cluster occurred after Patient A had had three hospital admissions for DFBI (Table 2). It is unclear why she initially started DFBI. Prior to this she had been living with an older female with BPD, who had a history of placing razor blades in her rectum and vagina, however had not swallowed items. From June 2013 the cluster of DFBI behaviour occurred with Patients B, C and D swallowing various objects. All four patients were aware of each other's hospital assessments.

Medical/Surgical Management: Initial management of Patient A was with endoscopy (episode 1) and endoscopy leading to gastrotomy (episode 2). Post-operative management was complicated by acting-out behaviour, and after discharge the patient re-opened her abdominal sutures with a pencil sharpener blade. A more conservative approach to surgical/medical management was adopted as further episodes occurred. At this time, endoscopies were still performed; however there was no surgery to recover swallowed items. Subsequently most patients 
Table 1. Published case reports of patients with personality disorders and DFBI.

\begin{tabular}{ccccc}
\hline Study & n & Female & BPD & Inpatient \\
\hline Present study & 4 & 4 & 4 & 2 \\
Reisner 2013 [9] & 3 & 3 & 3 & 2 \\
Rashid 2011 [10] & 1 & 1 & 1 & 1 \\
Frei-Lanter 2012 [4] & 1 & 1 & 1 & $?$ \\
Poynter 2011 [1] & 1 & 1 & 1 & $?$ \\
Gitlin 2007 [3] & 5 & 4 & 4 & $?$ \\
Soong 1990 [11] & 1 & 0 & $?$ & $?$ \\
James 1982 [12] & 3 & 1 & $14(93 \%)$ & $6(67 \%)$ \\
Total (\%) & 19 & $15(79 \%)$ & & $?$ \\
\hline
\end{tabular}

Table 2. Timing of DFBI incidents in the four patients (A-D), between Dec 2012 and Sep 2013.

\begin{tabular}{|c|c|c|c|c|c|c|c|c|c|c|}
\hline $\mathrm{Pt}$ & Dec & Jan & Feb & Mar & Apr & May & Jun & Jul & Aug & Sep \\
\hline A & 27 & & & & 23 & 1231 & $\begin{array}{ll}7 & 18\end{array}$ & 30 & & \\
\hline B & & & & & & & $\begin{array}{ll}3 & 13\end{array}$ & & & \\
\hline $\mathrm{C}$ & & & & & & & 23 & & & \\
\hline $\mathrm{D}$ & & & & & & & & & 19 & 7 \\
\hline
\end{tabular}

were just observed, and patients were discharged from hospital earlier. This approach has been successful to date, with no complications identified, and all objects not removed endoscopically being passed uneventfully.

Psychological Management: Initial management of Patient A was restrictive, with intensive nursing supervision and use of the Mental Health Act. This appeared be counterproductive, with further episodes of self-harm, and increased anxiety in nursing and medical staff caring for her post-operatively. Subsequent approaches avoided restrictive interventions, and focused instead on identification of negative emotions and enhancing coping mechanisms.

Liaison between Services: Another important aspect to management of patient care involved intensive interdisciplinary support and education. Anxiety and frustration amongst medical/surgical staff was a common response to hospitalization of patients post-DFBI, with the potential for restrictive ward management (e.g. 1-on-1 nursing, use of the Mental Health Act, restraints). The Consult/Liaison Psychiatry team played an important role in educating and supporting medical and surgical teams.

\section{Discussion}

Although there are relatively few published case reports of DFBI in patients with personality disorders $(n=15)$, it may be more prevalent than the paucity of case reports would suggest [3] [4]. Published case reports of DFBI indicate a predominantly young female population, mostly with BPD, with extensive histories of self-harm, and high rates of psychiatric hospitalization (Table 1). The 4 patients reported here fit this demographic profile. Uniquely, there appears to be clustering of DFBI, with Patients B, C and D engaging in this behaviour after Patient A had been hospitalized several times for swallowing objects. Although DFBI was associated with precipitants (intoxication, experiencing strong emotions), none were identified in 5/12 episodes.

The types of objects swallowed were generally sharp, and were not dissimilar to those typically used for cutting. This contrasts with characteristics of foreign bodies swallowed in a US population of predominantly psychiatric inpatients, where glass and razor blades made up only 3\% of 262 objects swallowed [2]. The proportion of patients with BPD in this study population was not reported.

Evidence-based guidelines are available for the acute medical and surgical management of DFBI [5] [6]. However no guidelines or studies exist on the appropriateness and timing of repeated endoscopies in patients with BPD who repeatedly ingest foreign objects [4]. Our experience gained with this DFBI cluster has led to the following strategy: 1) obtain x-rays to determine the location and nature of the swallowed objects; 2) if these are in the stomach, and $<6 \mathrm{~cm}$ in length, patients can be observed outside of hospital, until the objects have been passed naturally [13]. This applies whether or not the objects are sharp. 
There is very little information on the psychiatric and psychological management of DFBI in patients with BPD [1] [3]. The evidence supporting the effectiveness of drug treatment for symptoms of BPD is weak [14], and given the apparent rarity of DFBI in BPD, the prospect of having controlled drug trials on DFBI is unlikely. There are well developed evidence-based psychological therapies for treatment of self-harming behaviour in BPD [15] [16]. It has been suggested that DFBI in patients with BPD is a means to managing intense negative emotions and/or to get social reinforcement, similar to motivations associated with other forms of self-harm [9]. Our approach to patients was to focus on identifying distress and enhancing coping mechanisms, and to work on enhancing self-efficacy and responsibility. We attempted to work within the least restrictive environment, and avoided using the Mental Health Act. This approach is not dissimilar to the non-coercive therapeutic relationship advocated by Linehan and others [15] [17], with the intention of developing alternative coping strategies for intense emotions, and to achieve social reinforcement in more appropriate ways.

Management of DFBI in BPD patients involved many medical disciplines, including the Emergency Department, Medical and Surgical inpatient services, and Consultation Liaison and inpatient psychiatry services. Early on in the DFBI cluster, a common reaction after hospitalization of these patients was anxiety and frustration amongst medical/surgical hospital staff, and a tendency to manage patients restrictively. The importance of managing staff countertransference has been emphasised previously [1], and relevant processes to achieve this include inter-disciplinary support, collaboration, liaison and education were essential, to develop management plans for inpatient and outpatient care.

\section{Conclusion}

DFBI in patients with BPD is probably more common than the few published case reports might suggest. Management of DFBI in patients with BPD requires high levels of interdisciplinary collaboration. In the cluster of DFBI incidents presented in this paper, effective management was achieved with conservative medical/surgihods and non-restrictive psychological approaches that enhanced patient self-efficacy.

\section{Competing Interests}

In the last 5 years Dr Glue has received research funding from Douglas Pharmaceuticals and Demerx Pharmaceuticals, and has attended an advisory board for Janssen. The other authors declare that they have no competing interests.

\section{Authors Contributions}

All authors were involved in data acquisition, and drafting and revising the manuscript. All authors read and approved the final manuscript.

\section{References}

[1] Poynter, B.A., Hunter, J.J., Coverdale, J.H. and Kempinsky, C.A. (2011) Hard to Swallow: A Systematic Review of Deliberate Foreign Body Ingestion. General Hospital Psychiatry, 33, 518-524. http://dx.doi.org/10.1016/j.genhosppsych.2011.06.011

[2] Palta, R., Sahota, A., Bemarki, A., Salama, P., Simpson, N. and Laine, L. (2009) Foreign-Body Ingestion: Characteristics and Outcomes in a Lower Socioeconomic Population with Predominantly Intentional Ingestion. Gastrointestinal Endoscopy, 69, 426-433. http://dx.doi.org/10.1016/j.gie.2008.05.072

[3] Gitlin, D.F., Caplan, J.P., Rogers, M.P., Avni-Barron, O., Braun, I. and Barsky, A.J. (2007) Foreign-Body Ingestion in Patients with Personality Disorders. Psychosomatics, 48, 162-166. http://dx.doi.org/10.1176/appi.psy.48.2.162

[4] Frei-Lanter, C.M., Vavricka, S.R., Kruger, T.H., et al. (2012) Endoscopy for Repeatedly Ingested Sharp Foreign Bodies in Patients with Borderline Personality Disorder: An International Survey. European Journal of Gastroenterology and Hepatology, 24, 793-797. http://dx.doi.org/10.1097/MEG.0b013e32835403d5

[5] Eisen, G.M., Baron, T.H., Dominitz, J.A., et al. (2002) Guideline for the Management of Ingested Foreign bodiEs. Gastrointestinal Endoscopy, 55, 802-806. http://dx.doi.org/10.1016/S0016-5107(02)70407-0

[6] Ikenberry, S.O., Jue, T.L., Anderson, M.A., et al. (2011) Management of Ingested Foreign Bodies and Food Impactions. Gastrointestinal Endoscopy, 73, 1085-1091. http://dx.doi.org/10.1016/j.gie.2010.11.010

[7] http://ethics.health.govt.nz/system/files/documents/pages/HDEC\%20scope\%20summary.pdf 
[8] American Psychiatric Association (2013) Diagnostic and Statistical Manual. 5th Edition, APA Press, Washington DC.

[9] Reisner, A.D., Bornovalova, M.A., Gordish, L., Baker, R.N., Smith, K.J. and Sexton, R.E. (2013) Ingestion of Foreign Objects as a Means on Non-Lethal Self-Injury. Personality Disorders, 4, 182-189. http://dx.doi.org/10.1037/a0027954

[10] Rashid, M. and Gosai, I. (2011) The Girl Who Swallows Knives: Uncontrollable Deliberate Self-Harm in a Teenage girl with Borderline Personality Disorder. BMJ Case Reports, 2011, bcr0720103136. http://dx.doi.org/10.1136/bcr.07.2010.3136

[11] Soong, C.V., Harvey, C. and Doherty, M. (1990) Self-Mutilating Behaviour and Deliberate Ingestion of Foreign Bodies. Ulster Medical Journal, 59, 213-216.

[12] James, A.H. and Allen-Mersh, T. (1982) Recognition and Management of Patients Who Repeatedly Swallow Foreign Bodies. Journal of the Royal Society of Medicine, 75, 107-110.

[13] Hendry, K., Glue, P., Hill, J., Schultz, M., Ingestion of Sharp Foreign Objects. A Case Series, Literature Review and Management Recommendation. Submitted manuscript.

[14] Lieb, K., Völlm, B., Rücker, G., Timmer, A. and Stoffers, J.M. (2010) Pharmacotherapy for Borderline Personality Disorder: Cochrane Systematic Review of Randomized Trials. British Journal of Psychiatry, 196, 4-12. http://dx.doi.org/10.1192/bjp.bp.108.062984

[15] Linehan, M.M. (1993) Cognitive-Behavioral Treatment of Borderline Personality Disorder. Guilford, New York.

[16] Stoffers, J.M., Völlm, B.A., Rücker, G., Timmer, A., Huband, N. and Lieb, K. (2012) Psychological Therapies for People with Borderline Personality Disorder. Cochrane Database of Systematic Reviews, 8, CD005652.

[17] Connors, R. (2000) Self Injury: Psychotherapy with People Who Engage in Self-Inflicted Violence. Jason Aronson, Northvale. 